\title{
Fragmentation, Reaction and Photodetachment of Mass Separated Cluster Ions
}

\author{
W. Begemann, S. Dreihöfer, G. Ganteför, H.R. Siekmann,
} K.H. Meiwes-Broer, and H.O. Lutz

Fakultät für Physik, Universität Bielefeld, D-4800 Bielefeld 1, Fed Rep. of Germany

Mass separated metal and semiconductor cluster ion beams have been produced by sputtering as well as laser evaporation. During and after the production process, sputtered clusters fragment at a high rate. Cooling of laser evaporated clusters in a He carrier gas results in stable configurations. The spontaneous fragmentation, fragmentation in collisions, chemical reactions with clusters, and photoelectron spectroscopy of cluster ions is the main topic of the present investigations.

\section{INTRODUCTION}

The interaction of free size-selected clusters with laser fields or collision partners allows insight into fundamental properties like electronic and geometrical structure, reactivity etc. There have been a number of studies involving neutral clusters in which it is difficult to unambiguously assign the collision partners. Working with cluster ions reduces this ambiguity because a defined cluster size can be selected; in addition, the charged products do not have to be ionized prior to product mass analysis.

The paper presents investigations of a variety of sputtered as well as laser-evaporated cluster ions $\left(A 1_{n}, S i_{n} C_{n}{ }^{\prime} W_{n}\right.$ $\left.\mathrm{Cu}_{n} F_{m}, \mathrm{Al}_{\mathrm{n}} \mathrm{F}_{\mathrm{m}}\right)$. After the study of sputtered clusters and their unimolecular decomposition in section I, section II describes the "reflectron" mass spectrometer as is used for fragment analysis. The results of collision-induced fragmentation of negatively and positively charged sputtered clusters, including first results of charge exchange, are given in the third chapter. In the last part the production of cold metal and compound cluster ions is described; in this section we also present the spectroscopy of photodetached electrons of negatively charged metal clusters. 
Sputtering of solid targets by high energy ion bombardment produces neutral as well as charged clusters. Several attempts have been made to describe the production process theoretically; two different growth models are discussed;

a) The whole cluster emerges from the surface; in this case the constituents must stem from neighbouring lattice places. /1/

b) Sputtered atoms from one collision cascade recombine above the surface in the vacuum. $/ 2 /$

In both cases the relative velocity of the combining atoms or molecules must be low so that the total energy of the nascent cluster is lower than its dissociation energy. None of the models has been uniquely favoured by experimental results so far; measurements of kinetic energy distributions of sputtered metal clusters show a decrease in width and mean energy with increasing number of atoms $\mathrm{n} / 3 /$. From this it has been concluded that the low energy part of the collision cascade is mainly responsible for cluster emission.

Experimental work on sputtering suffers from the fact that the produced species is internally excited and shows a strong tendency to fragment. In order to shed more light on this spontaneous behaviour we first investigated the unimolecular decomposition of sputtered metal and silicon cluster ions. The sputtered ion beam apparatus is described in detail elsewhere 14/. In short, the experiments are performed in a five-stage differentially pumped UHV machine (see Fig. 1) with pressures of $<10^{-8}$ mbar (base pressure) and $<10^{-7}$ mbar (during sputtering) inside the target chamber. Under these conditions the unimolecular decay is not disturbed by collisional relaxation or target contamination. Double differential pumping between target and scattering chamber turns out to be a prerequisite for chemical reaction experiments on sputtered clusters: A contamination of the sputter target by e.g. a partial pressure of only approximatively $1 \times 10^{-7}$ mbar oxygen changes the cluster ion production conditions severely so that the sputtered material contains a considerable amount of metal oxides. These oxides might partly be transmitted by the mass separator; in this case a clear distinction between gas-phase reaction products and sputtered oxides would be impossible. 


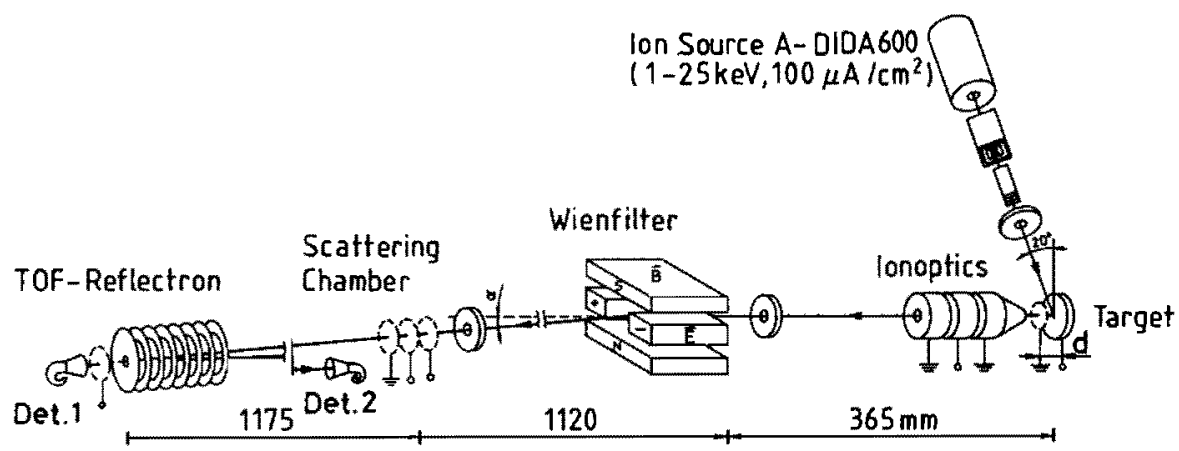

Fig. 1 . Ion beam apparatus for the investigation of sputtered cluster ions

Neutral and charged clusters are produced by about $20 \mathrm{keV}$ $\mathrm{Xe}^{+}$ion bombardment of thick targets. The cluster ions are then accelerated from the floated target $\left(v_{a c c}=500 \ldots 4000 \mathrm{~V}\right.$, typically $1800 \mathrm{v})$ and focussed through a wien filter into the scattering chamber.

A time-of-flight "reflectron" serves mainly for the analysis of collision-induced fragments or chemical reaction products and is described in chapter III. For the investigation of the unimolecular decomposition detector 1 (see Fig.1) is utilized with a repeller grid energy analyzer mounted in front. This setup defines a time window in which the decay is measured: After acceleration over a preselected distance $d(1.7$ or $18.5 \mathrm{~mm})$ the cluster ions undergo velocity selection while passing the wien filter (COLUTRoN $600 \mathrm{~B}$ ). Only those clusters (of specific mass) are selected which do not fragment for at least a time $t_{0}$ after they were produced at or immediately in front of the target, $t_{0}$ being the time needed by the cluster beam to reach its final energy; it varies, e.g., (for $\mathrm{V}_{\mathrm{acc}}=1.3 \mathrm{kV}$ ) between $35 \mathrm{~ns}\left(\mathrm{Al}^{+}\right.$, $\mathrm{d}=1.7 \mathrm{~mm})$ and $2.7 \mathrm{\mu s}\left(\mathrm{cu}_{21}^{+} \mathrm{d}=18.5 \mathrm{~mm}\right)$. All particles which fragment before acceleration has been completed have different velocities and are deflected out of the beam by the wien filter. Fragments being produced after acceleration keep the parent's velocity and will be transmitted. Therefore, the ion beam just in front of the detector contains the parent ions and their decay products which arise in a time window which is opened at $t_{0}$ and closed when the particles reach the detector. The repeller-grid in front of the multiplier separates fragment ions from the clus- 
ter ion beam. Intensity steps appear at voltages sufficient for repelling the fragments and reveal uniquely the unimolecular decay into defined fragments for given time windows.

\section{Wien Filter Mass Spectra and Decay Rates}

The ion beam composition is studied either by chopping the cluster beam and performing a time-of-flight analysis in the reflectron, or by scanning the deflection voltage of the wien filter and monitoring the transmitted ion current.

The second method yields spectra with lower resolution but higher intensity. Fig. 2 shows as examples three wien filter spectra of negatively charged aluminium, silicon and copper clusters.

Mass selected cluster ion beams can thus be used for further investigation. The mass range available in this experiment depends on the maximum size of clusters produced by sputtering and on the wien filter resolution (which could be increased by inserting an energy filter between target and wien filter).

For unimolecular decay studies the ion beam current for the mass of interest is monitored by detector 1 (Fig.1); scanning
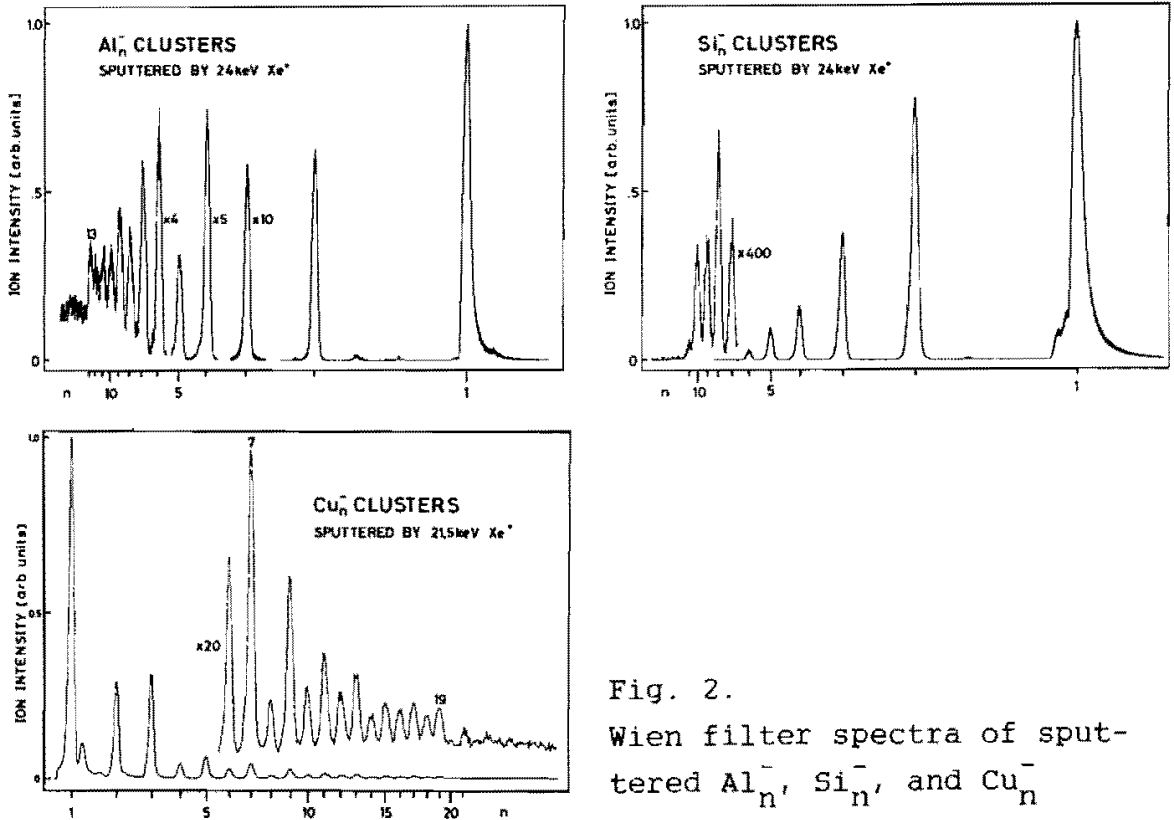

Fig. 2 .

Wien filter spectra of sputtered $\mathrm{Al}_{\mathrm{n}^{\prime}}^{-} \mathrm{Si}_{\mathrm{n}^{\prime}}^{-}$and $\mathrm{Cu}_{\mathrm{n}}^{-}$ 


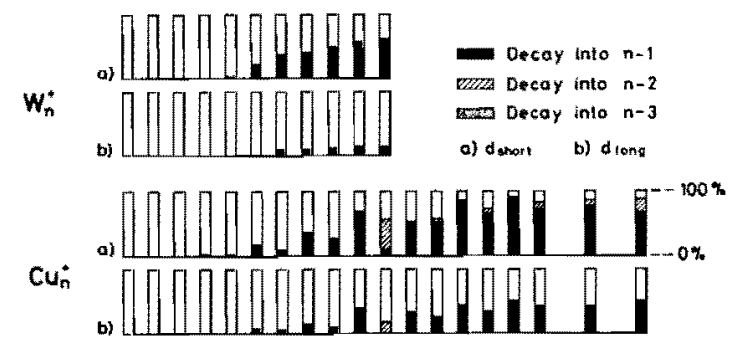

$\operatorname{Sin}$

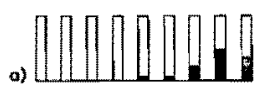

Fig. 3 .

Decay (in $\%$ ) of sputtered $A I_{n^{\prime}}^{+} \quad S i_{n^{\prime}}^{+} \quad C u_{n}^{+}$and $w_{n}^{+}$into positively charged fragments for the long $\left(d_{\text {long }}=18.5\right.$ $\mathrm{mm})$ and short $\left(\mathrm{d}_{\text {short }}=1.7\right.$ $\mathrm{mm}$ ) acceleration distances. Statistical error $\pm 3.5 \%$

$A l_{n}^{*}$

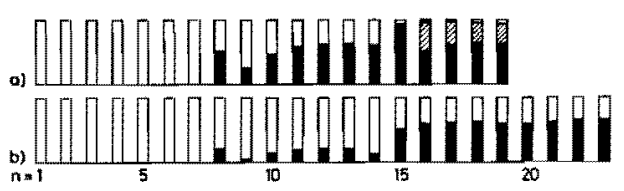

the repeller grid voltage results in intensity steps and gives the decay rates which are shown in Fig. 3 for $\mathrm{Al}_{n}^{+}, \mathrm{Si}_{\mathrm{n}^{\prime}}^{+} \mathrm{Cu}_{\mathrm{n}}^{+}$and $w_{n}^{+}$.

In all cases small cluster ions with $n \leq 3$ are practically stable within the experiment's time windows $\Delta t$. Strong decomposition is observed for larger $n$, increasing with $n$. When $t_{0}$ is shifted towards the production event ( $d_{\text {long }} \rightarrow d_{\text {short }}$ ) the decay probability also rises. A variation of $\Delta t$ by enlarging the total drift length from 1.5 to $2.5 \mathrm{~m}$ affects the outcome of the experiment only insignificantly. The dominating charged decay product is the (n-1)-fragment (black bars in Fig. 3), (n-2)-docomposition occurs in some cases. (n-3)-fragments are observed for $\mathrm{Si}_{9}^{+}$whose main fragment is $\mathrm{Si}_{6}^{+}$. This decomposition seems to proceed via fission of $\mathrm{Si}_{9}^{+}$since no significant decay of $\mathrm{Si}_{8}^{+}$to $\mathrm{Si}_{6}^{+}$is observed. For metal clusters there is no hint of a fission process: After some initial cooling time $t_{0}$, sputtered metal cluster ions apparently loose further excess energy mainly by evaporation of single neutral atoms. If $t_{0}$ is reduced $\left(d_{\text {short }}\right)$, two-atom decomposition becomes more prominent.

The decay probabilities for $\mathrm{Cu}_{n}^{+}$have been calculated by KLOTS /5/: An "evaporative ensemble", defined by a collection of excited cluster ions, starts to shed both matter and energy by evaporation of neutral atoms. Thermodynamic evaporation combined 
with classical unimolecular decay theory yields decay rates which are in good agreement with the mean $n$-dependence of the measured $\mathrm{Cu}_{\mathrm{n}}^{+}$decomposition for $\mathrm{n} \geq 7$. These findings again support the assumption that excited copper cluster ions evaporate single neutral atoms, cool down and gain stability.

A number of consequences arise from the results given above. For a thorough discussion we refer to earlier publications $/ 4,6 /$; only a few aspects will be given here:

a) Mass spectra of sputtered cluster ions are qualitatively mirror images of the decay probabilities. The intensity distributions turn out to be a result of decomposition cascades in which clusters with high stability survive and show up more abundantly. Therefore, the shape of a mass spectrum is a function of the time between cluster production and detection. similar observations have been made by ENS et al. on sputtered alkali halide clustex ions $/ 7 /$.

b) The investigation of the sputtering process will lead to unambiguous results only when the time dependence of metastable decomposition is properly taken into account. In other words: The clusters whose kinetic energy or internal energies are investigated some ns or us after theix production might not be those which have been born on the target surface.

c) Mass spectra after ionization (by electrons or light) of cold neutral metal clusters often show fine structure which is similar to those of sputtered cluster ions. The spectra of cold metal cluster ions emerging directly from a source without post-ionization (c.f. chapter IV), however, display no fine structure. We conclude that even "careful" ionization may lead to pronounced fragmentation resulting in the obsexved fine structure.

d) The metastable decompositions of metal and silicon clusters cease after several us. It is possible to investigate stable sputtered cluster ions when they have had a chance to cool down by evaporation.

e) The unimolecular decay by evaporation of neutral atoms is a quite general behaviour also of non-metal cluster ions: Measurements of MARK et al. /8/ and CASTLEMAN et al. /9/ show a preferred $(n-1)$ decomposition for raxe gas and for hydrogen-bonded systems. 
II. A TIME-OF-FLIGHT REFLECTRON FOR MASS ANALYSIS OF FRAGMENTS AND REACTION PRODUCTS

A time-of-flight ( $T O F$ ) reflectron is a mass spectrometer with bent drift line (see Fig. 1). This instrument was developed by MAMYRIN et al. /10/ and has mainly been used because of its high mass resolving power in laser ionization experiments of molecules /11/. Now the TOF reflectron becomes of growing importance for the spectroscopy and fragment analysis of clusters $/ 4,12-14 /$.

In the experiment described in chapter I we have the special situation that a continous beam of charged clusters has to be analyzed, possibly together with charged fragments produced by collisions, light or chemical reaction. For time-of-flight analysis, such a continuous cluster beam must be pulsed, which leads to ion bunches with considerable spread in translational energy. A TOF reflectron is able to compensate for this energy width; we have studied this compensation both theoretically and experimentally. Mass spectra with improved resolution are presented and discussed. In addition, the usefulness of the reflectron for fragment analysis is tested by a computer simulation of decomposing cluster ions as well as by a collision induced fragmentation experiment.

\section{Mass Spectroscopy in the TOF Reflectron}

Ion pulses of defined mass pass the field-free drift region and are rapidly decelerated in the first retarding potential $U_{1}$, see Fig. 4.

They then enter the reflecting field $\left(\mathrm{U}_{2}-\mathrm{U}_{1}\right)$ with low velocity, where their differences in flight time are corrected. The

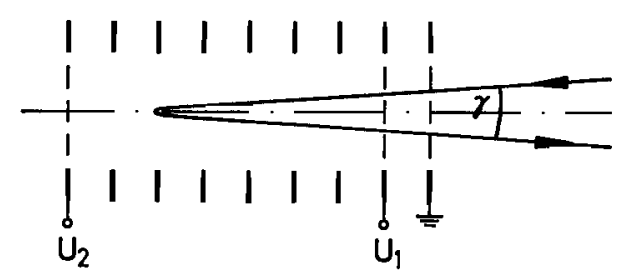

Fig. 4. Retarding $\left(\mathrm{U}_{1}\right)$ and reflecting $\left(\mathrm{U}_{2}\right)$ voltages in a TOF reflectron 
reflected ion beam (inclined at an angle of $\gamma=3.5^{\circ}$ to the incident beam) is detected by a tandem channel plate.

For the calculation of the total flight time (from pulse formation to detection) the flight times in each stage (two-stage acceleration, field-free drift, retardation, reflection, reacceleration and second field-free drift) are added and calculated as function of the voltage ratio $v_{1} /\left(U_{2}-U_{1}\right)$, the reflecting voltage $\mathrm{U}_{2}$ and the ion energy $E_{i o n}$. The results for an optimized ratio $\mathrm{U}_{1} /\left(\mathrm{U}_{2}-\mathrm{U}_{1}\right)=2$ are depicted as contour lines in Fig. 5.

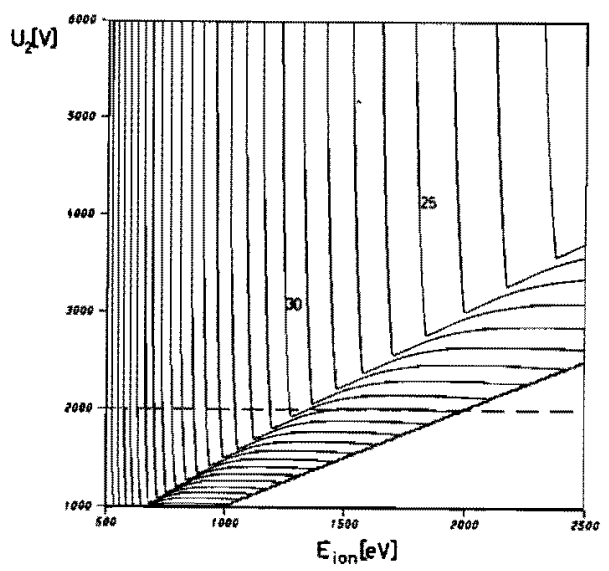

Fig. 5.

Calculated contour map of the flight time (in us) from start to detection in the TOF reflectron for $\mathrm{Cu}^{+}$, as function of the reflection voltage $\mathrm{U}_{2}$ and the ion energy $E_{\text {ion }}$. The spacing between the contour lines is $1 \mathrm{\mu s}$

Compensation of energy spread in the ion bunches occurs when the contour lines are parallel to the $x$-axis. As an example for $\mathrm{U}_{2}=2000 \mathrm{~V}$ (a typical value in these experiments, dashed line in Fig. 5), ions with $E_{\text {ion }}$ ranging from 1400 to $1900 \mathrm{eV}$ need the same flight time of $31 \pm 0.1 \mu \mathrm{s}$. Consequently, ion bunches with an energy width of up to $500 \mathrm{eV}$ should be focussed to a $0.1 \mathrm{\mu s}$ bunch.

As experimental verification, an $\mathrm{Al}^{+}$ion bunch with an artificially large energy width of $400 \mathrm{eV}$ is produced. Fig. 6 shows the resulting ToF spectra in forward direction (detector 1) and reflected (detector 2 ).

The broad peak (Fig.6a) is focussed by the TOF reflectron into a line with 0.3 us width for optimized reflection voltages (Fig.6b). In practical use, the energy spread is much smaller; for the analysis of the sputtered cluster ion beam composition a number of different pulse-forming methods (including single or 


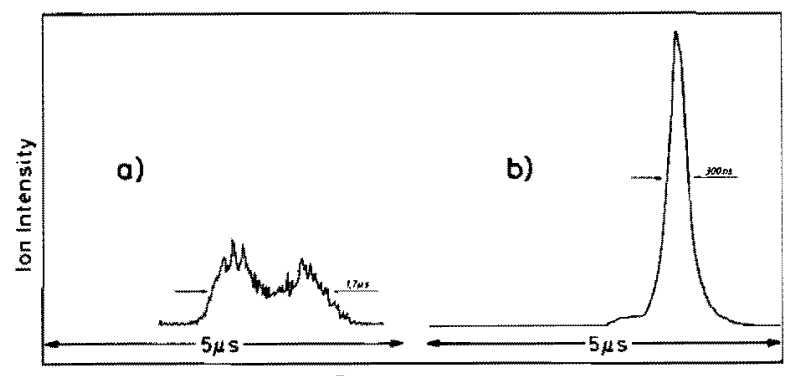

TIME OF FLIGHT

Fig. 6. TOF spectra of $\mathrm{Al}^{+}$bunches having an energy width of 400 $\mathrm{eV}$, recorded in a) forward direction and b) reflected

double grid switching, chopping combined with grid switching etc.) were used. As examples Fig. 7 shows such mass spectra obtained by chopping the ion beam at the entrance of the scattering chamber.

Note that the spectra show the beam composition several us after the sputtering process, i.e. when the clusters have reached the scattering chamber. At this position the metastable decomposition has practically ceased. The spectra are, there-
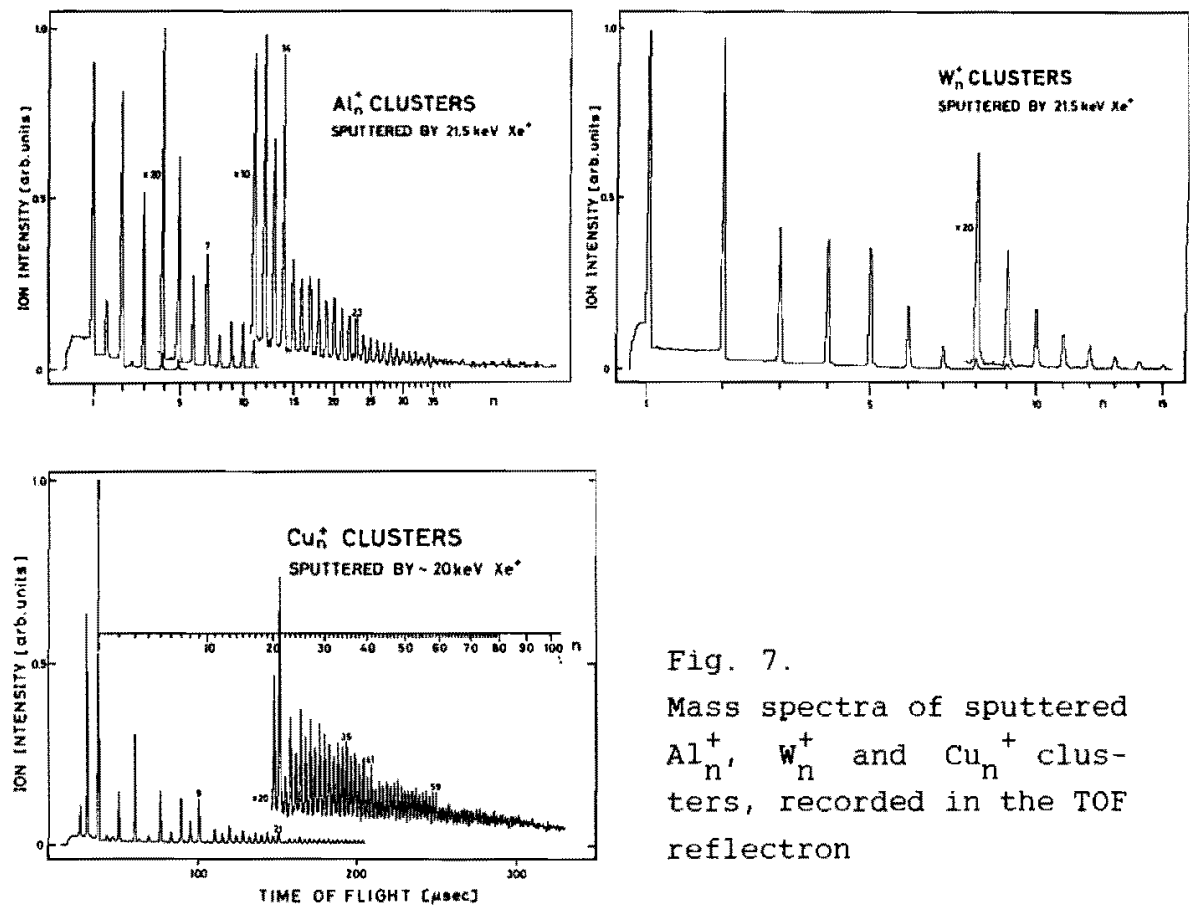

Fig. 7.

Mass spectra of sputtered $\mathrm{Al}_{\mathrm{n}^{\prime}}^{+}, \mathrm{W}_{\mathrm{n}}^{+}$and $\mathrm{Cu}_{\mathrm{n}}^{+}$clusters, recorded in the TOF reflectron 
fore, the result of a number of decay processes and reveal the thermodynamic stability of the species as has been discussed in the previous section. This is especially obvious in the $\mathrm{Al}_{n}^{+}$and $\mathrm{Cu}_{n}^{+}$spectra where pronounced fine structures occur: Aluminium shows enhanced intensities for $n=7,14$ and 23; the copper spectrum is governed by a pronounced even-odd intensity alternation which can be attributed to the increased stability of clusters possessing spin-paired binding electrons. In addition pronounced lines arise at $n=9,21,35,41,59$ and are partly followed by strong intensity drops.

Most of these intense lines correspond to the "shell closings" as has been discussed by HERMANN et al. /15/, ECKARDT /16/, KNIGHT et al. $/ 17 /$ and CHou et al. /18/. This model regards a metal cluster as a conducting drop in which the positive charge of the $n$ atom cores is homogeneously distributed. The valence electrons are then filled into this potential well so that a neutral "jellium" is formed. As a consequence of the spherical symmetry the electronic eigenstates have the same degeneracies as those of a hydrogen atom. The spatially extended positive charge distribution, however, causes a different ordering pattern. The quantum mechanics of this structureless problem has been worked out in different approximations $/ 16,18 /$. The resulting closed-shell clusters (containing $2,8,18,20,34,40$, 58 ... electrons) have enhanced relative binding energies. Although these calculations mainly concern alkali metal clusters, nearly the whole rich structure in the spectra of sputtered positively and negatively charged copper clusters can be explained (except the even-odd-alternation). Even for $\mathrm{Al}_{\mathrm{n}}^{+}$a part of intensity abnormalities match with the shell model. Sputtered cluster ion mass spectra taken by other authors with different methods also show pronounced fine structures for a number of metals 119,20\%. A strong correlation between the shapes of the spectra and shell closings is observed.

\section{Fraqment Analysis in the ToF reflectron}

The rof reflectron can be operated in several modes: For the investigation of cluster collisions at variable ion energies, the beam is decelerated into a cell, crosses the gas target and is reaccelerated for TOF analysis. In this mode the instrument 
works as a mass spectrometer. In another mode cluster ion pulses hit without deceleration onto the gas target so that the parent ions as well as their fragments have nearly the same velocity. According to the fragment/parent mass ratio the fragments have lower energy, but reach the reflector at the same time as the parents. Since the ions penetration depth into the reflecting field is a function of their energy, their time of arrival at the detector is a function of their mass.

For a thorough understanding of the fragment mass spectra computer simulations of the decay of copper cluster ions were performed. Fig. 8 shows as an example such a spectrum for $\mathrm{Cu}_{7}^{+}$.

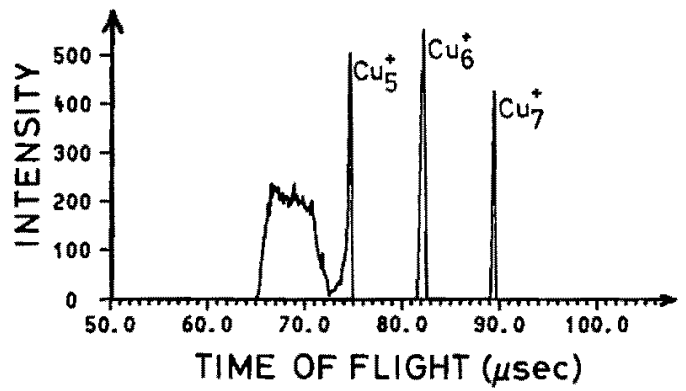

Fig. 8. Simulated fragment spectrum of the decay of $\mathrm{Cu}_{7}{ }^{+}$in the field-free drift tube of a TOF reflectron

It is supposed in the calculations that all fragments $\mathrm{Cu}^{+} \ldots \mathrm{Cu}_{6}^{+}$, as well as the parent ions, appear with equal strength. The parent ion energy (1800 ev) has an assumed width of $10 \mathrm{ev}$. Note that the parent mass has reduced intensity since the reflecting voltage is chosen to be $U_{2}=1800 \mathrm{~V}$, thus preventing part of the parent ions from being reflected. Only the fragment masses $\mathrm{Cu}_{5}^{+}$and $\mathrm{Cu}_{6}^{+}$are resolved as their energy is sufficient to overcome the retarding potential $U_{1}$, see Fig. 4. The smaller fragments $\left(n(5)\right.$ are reflected at $U_{1}$ and appear as a broad unresolved line between $65 \mathrm{\mu s}$ and $72 \mu \mathrm{s}$. Lowering $U_{1}$ and $U_{2}$ (for high resolution the voltage ratio has to be kept constant, i.e. $\left.\mathrm{U}_{1} /\left(\mathrm{U}_{2}-\mathrm{U}_{1}\right)=2\right)$ the contributions of the smaller fragments will be resolved whereas the large masses vanish since their energy is now higher than $\mathrm{eU}_{2}$.

In an experimental verification, $1.8 \mathrm{keV} \mathrm{Cu}_{9}^{+}$cluster pulses hit onto a $5 \times 10^{-5}$ mbar argon gas. The resulting fragment spectra 


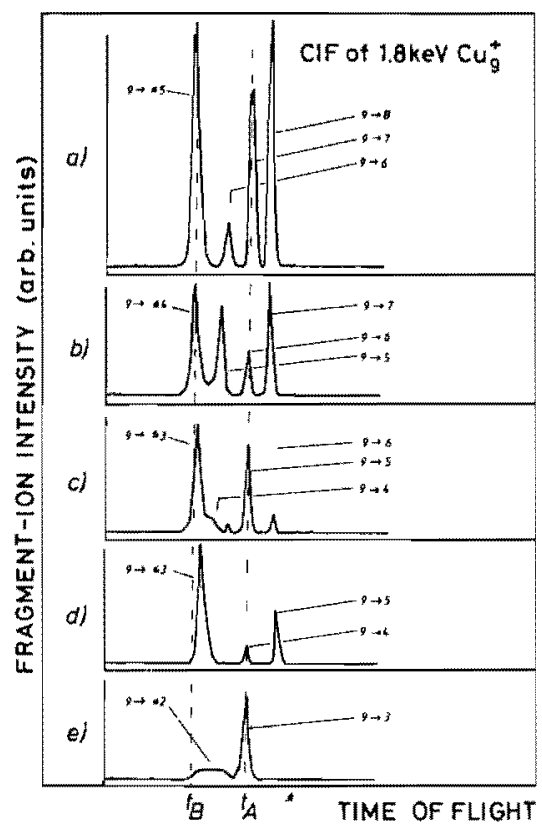

Fig. 9.

TOF reflectron fragment spectra of $1.8 \mathrm{keV} \mathrm{Cu}_{9}^{+}$collisions in $5 \times 10^{-5}$ mbar argon. Successively lowering the reflecting voltages (a...e) makes different fragment lines appear at position $t_{A}$

are given in Fig. 9 as a function of the reflecting voltage $\mathrm{U}_{2}$ (a...e). Parts a) and b) show spectra in which large fragments are resolved, whereas all contributions from the small fragments form a broad line near $t_{B}$. For an intensity analysis the reflecting voltages are tuned so that the fragment line of interest is shifted to a defined position $t_{A}$ in the ToF spectra.

The lines corresponding to smaller fragments are successively extracted out of the unresolved peak near $t_{B}$ and integrated when they reach $t_{A}$. With this method "collision induced fragmentation" cross sections of mass selected metal and silicon ions can be determined.

III. COLLISION INDUCED FRAGMENTATION AND NEUTRALIZATION OF METAL AND SILICON CLUSTER IONS

Collisions of (hydrogen) cluster ions in a low pressure gas target have first been investigated by VAN LUMIG et al. /21/. They called the resulting break up of the clusters "fragmentation". Fragmentation of metal dimers was investigated by ERVIN et al. /22/ and yielded bond energies. Collision induced frag- 
mentation (CIF) of metal cluster ions (consisting of more than two atoms) was first carried out by our group $/ 4 /$. Recently, JARROLD et al. /23/, HANLEY et al. /24/ and we /25/ studied the fragmentation behaviour of $\mathrm{Al}_{\mathrm{n}}^{+}$in rare gas targets using different collision energies.

In this paper we discuss the CIF of positively and negatively charged metal and semiconductor clusters. Monodispersed sputtered cluster ions impinge onto the $1 \ldots 10 \times 10^{-5}$ mbar gas target. CIF cross sections ( $a_{\text {frag }}$ ) are obtained without deceleration of the $1.8 \mathrm{keV}$ cluster ion beam, i.e. the center-of-mass collision energies $E_{C M}$ vary e.g. for $\mathrm{Cu}_{n} \rightarrow \operatorname{Ar}$ from $117 \ldots 431 \mathrm{eV}$, with $n=9 \ldots 2$. It turns out that in this energy region ${ }_{\text {frag }}$ is independent of $\mathrm{E}_{\mathrm{CM}}$. For product analysis alternatively two different methods are applied:

a) Surviving parent ions as well as charged fragments undergo mass analysis in a time-of-flight reflectron (see chapter II); the fragments are separated from the parents and produce resolved lines in the spectrum.

b) A repeller grid energy analyzer separates CIF products from surviving parent clusters. This setup is also utilized to study the charge exchange of negative clusters in a gas target.

Experimental raw data are relative fragment intensities resulting from defined parent cluster ions as function of gas

CF of $1.8 \mathrm{keV} \mathrm{Al}$

$$
\text { 5. } 0^{-4} \text { mbar at }
$$

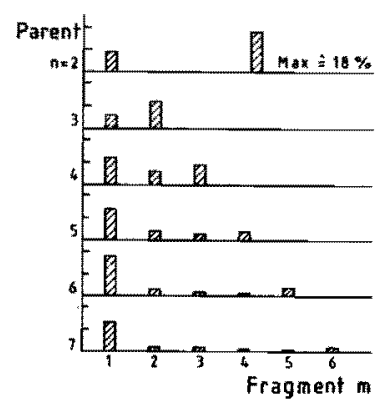

CIF of $9.8 \mathrm{keV} \mathrm{Al}_{\mathrm{n}}^{-}$

5. 10-5

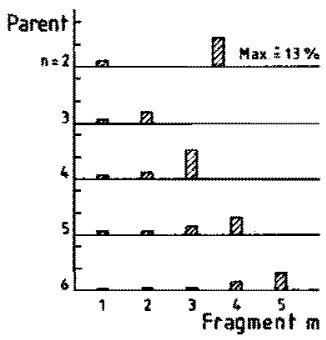

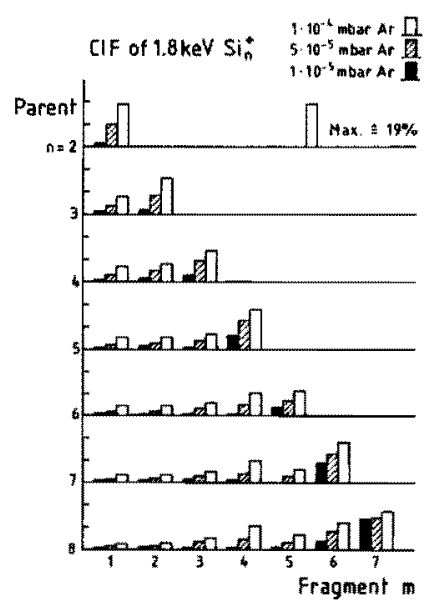

Fig. 10. Relative fragment intensities of cluster ion collisions in argon gas targets. The values are normalized to the intensity of all transmitted ions 
pressure. Fig. 10 shows such fragment patterns for $\mathrm{Al}_{\mathrm{n}^{\prime}}^{+} \mathrm{Al}_{\mathrm{n}}^{-}$and $\mathrm{Si}_{\mathrm{n}}^{+}$colljisjons in argon.

A dominant charged fragment of $\mathrm{Al}_{\mathrm{n}}^{+}$is $\mathrm{Al}^{+}$, i.e. the clusters lose one constituent, the charge remaining on the small fragment. Similar observations have been made by other authors where $E_{\mathrm{CM}}$ was reduced to $\leqslant 5 \mathrm{eV} / 23,24 /$. CIF of negatively charged aluminium clusters proceeds also mainly by the loss of one constituent, but now the charge remains on the large fragment. For $S i_{n}^{+} \rightarrow$ Ar also mainly $(n-1,2)$ fragments dominate. The same holds true in CIF of all other positively charged metal cluster ions we studied so far,like $\mathrm{Cu}_{n^{\prime}}^{+} \mathrm{w}_{n}^{+}$and $\mathrm{MO}_{n}^{+} / 4 /$. The fine structure of the CIF patterns, of course, is different for these systems. The extraordinary behaviour of $\mathrm{Al}_{n}{ }^{+}$might be due to the fact that - in contrast to other metal clusters - the ionization potential of $\mathrm{Al}_{\mathrm{n}}$ increases with $\mathrm{n}$ as has been calculated by UPTON $/ 26 /$.

For the determination of CIF cross sections the pressure dependence has to be taken into account (see Fig.10): In the case of, e.g., $\mathrm{Si}_{4}^{+}$, the patterns for the different pressures remain roughly unchanged. The $\mathrm{si}_{8}^{+}$pattern, however, shows a strong dependence on the gas pressure, thus indicating an influence of secondary collisions. Therefore, we calculate first the apparent cross sections $a_{\text {app }}$ by means of the fragment intensity sums which result from defined parent ions $\mathrm{n}$, for a set of different pressures:

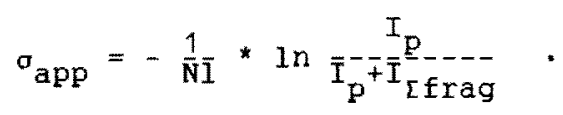

$N$ gives the gas density, 1 the collision chamber length, $I_{p}$ and $I_{\text {Ifrag }}$ are the intensities of the transmitted parent ions and the fragments, respectively. With this method the influence of the cluster beam absorption (scattering, neutralization), which turns out to be a strong function of $n$, is minimized: The signal is normalized to $I_{p}+I_{\text {Ifrag' }} i . e$. to the sum of all transmitted ions.

For larger clusters, oapp varies dramatically with $n$. A linear extrapolation of opp to zero pressure gives the total CIF cross sections $a_{\varepsilon \mathrm{mn}}$ as shown in Fig. $11 \mathrm{a}, \mathrm{b}$ for silicon and copper. 

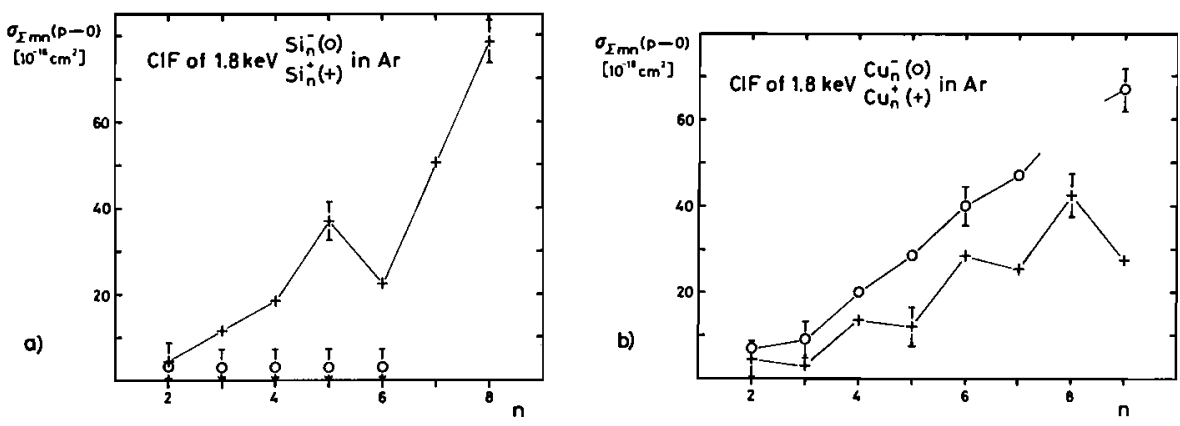

Fig. 11. Total CIF cross sections $\sigma_{\left[m n^{\prime}\right.}$ i.e. the cross section for fragmentation of an $n$-cluster into all m fragments.

a) silicon in argon. The values for $\mathrm{Si}_{\mathrm{n}}^{-}$are less than $5 \times 10^{-16} \mathrm{~cm}^{2}$, for all $\mathrm{n}$.

b) Copper in argon. The role of the charge is obvious

$\sigma_{\text {Imr }}$ shows for $\mathrm{Si}_{\mathrm{n}}^{+}$a nearly exponential rise with $\mathrm{n}$. superimposed is a pronounced minimum for $\mathrm{Si}_{6}^{+}$, being in coincidence with the enhanced binding energy as has been calculated previously $127 \%$. The increase of $\sigma_{\text {[mn }}$ with $\mathrm{n}$ is much steeper than for all metals which have been investigated so far. As an example Fig.11b gives the results for cationic and anionic copper clusters: $\mathrm{Cu}_{\mathrm{n}}^{+}$shows a marked even-odd variation of ${ }_{\Sigma \mathrm{mn}}$; this behaviour is a consequence of the enhanced binding energies of spin-paired metal clusters. The oscillations are nearly washed out for $\mathrm{Cu}_{n}^{-}$. In a separate experiment the transmission of copper cluster ions through an argon gas target has been measured to be practically independent of the cluster charge; thus the geometrical cross sections are not strongly dependent on the sign of the charge. Therefore, we conclude that the differences in $\sigma_{[\mathrm{mn}}$ are mainly a result of the different binding energies.

For negatively charged silicon clusters ${ }_{[\mathrm{mn}}$ is lower than $5 \times 10^{-16} \mathrm{~cm}^{2}$ for all $\mathrm{n}$, thus being in complete contrast to all metals studied so far. The absorption, however, turns out to be significantly larger than that of positively charged silicon clusters. This is a first hint of an effective neutralization of $\mathrm{Si}_{\mathrm{n}}^{-}$in $\mathrm{Ar}$. In the next step a repelling voltage is applied in front of the detector 1 in order to directly extract the contribution of neutral particles in the beam. As an example Fig.12 


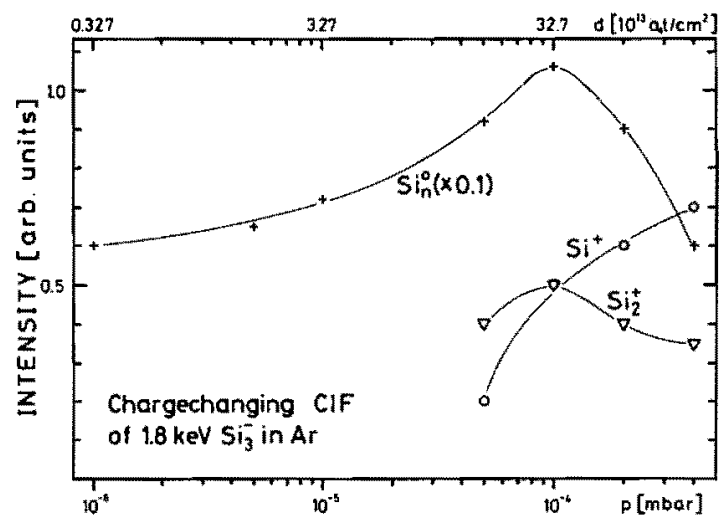

Fig. 12. Neutralisation and re-ionization of $5 i_{3}$ in $\mathrm{Ar}$ as function of gas pressure $p$, or thickness d. upper curve: Intensity of neutral particles. Lower curves: Positive ion signal after collisions of neqatively charged $\mathrm{Si}_{3}$ with the Ar target

gives the neutral particle intensity after collisions of $1.8 \mathrm{keV}$ $\mathrm{Si}_{3}^{-}$in Ar.

The neutral particle intensity rises smoothly with $p$ to a maximum at $1 \times 10^{-4}$ mbar, followed by a fast drop. At the same time the neutrals are ionized to $\mathrm{Si}_{2}{ }^{+}$in secondary collisions. Further pressure increase leads to fragmentation of $\mathrm{Si}_{2}^{+}$and ends up in the production of $\mathrm{si}^{+}$. For all $n$ studied so far the largest ionized clusters are the (n-1)-mers. With this crude method (neutralization and subsequent ionization in the argon gas target) it is not uniquely proved that the charge exchange of $\mathrm{Si}_{\mathrm{n}}^{-}$ leads to monodispersed neutral beams. But from the comparison of the CIF and transmission behaviour we find that the cross section for neutralization exceeds $a_{\Sigma m n}$ by about a factor of five to ten. This makes $5 i_{n}^{-}$a promising candidate for the production of mass selected neutral clusters.

IV. COLD METAL AND COMPOUND CLUSTER IONS: PRODUCTION AND PHOTO-

\section{ELECTRON SPECTROSCOPY}

The experiments described above have been performed on sputtered, i.e. excited cluster ions. To shed more light on the role of the cluster's temperature we also produce cluster ions in a pulsed laser vaporization source. Differences between such 
cold clusters and clusters prepared by other methods will be discussed. In addition, first results on the spectroscopy of photoelectrons of these species are presented.

\section{Cold Cluster Ion Beam Apparatus}

The laser vaporization source is similar to the standard technique of laser vaporization of a target rod of the desired metal mounted in a pulsed, high pressure supersonic nozzle $128,29 /$. Different lines of an excimer laser have been used to vaporize the metal, the resulting plasma being flushed out by a pulse of $\approx 1$ atm. helium or neon through a channel of variable length into high vacuum. We do not use a post-ionizer but study the charged clusters as they emerge from the source. In order to avoid any disturbance of the supersonic expansion, the area between nozzle and skimmer is carefully shielded from electric fields.

The ion beam composition is analyzed in a time-of-flight mass spectrometer which works collinearly to the source direction as shown in Fig.13. Appropriate switching of the accelerator entrance grid allows short ion pulses to drift into the starting area and to be accelerated in two stages for optimum time resolution. After having passed the ion optics the clusters are detected in a channeltron.

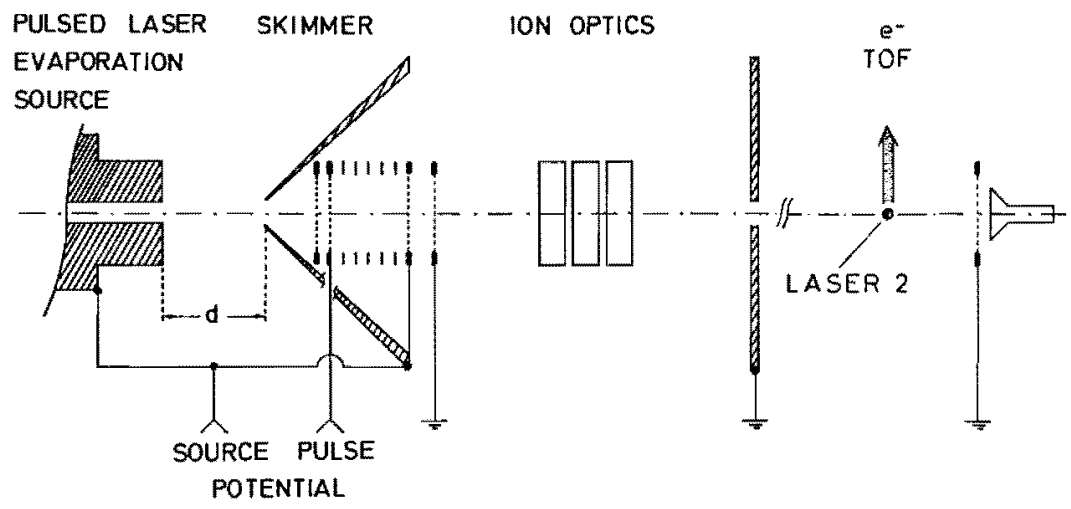

Fig. 13. Experimental setup for the investigation of cluster ions emerging from a laser evaporation source 
For the spectroscopy of photoelectrons a second (excimer) laser is used. Its delay is tuned in order to be in coincidence with the arrival of a defined ion bunch in the starting area of a TOF electron spectrometer. Without further acceleration the electrons start in a strongly divergent magnetic field and are adiabatically guided into a weak field drift tube $/ 30 /$.

\section{Spectra of cold cluster Ions}

Fig. 14 shows as examples the mass spectra of $\mathrm{Cu}_{\mathrm{n}}^{+}$and $\mathrm{Al}_{\mathrm{n}}^{-}$ emerging from the source without post-ionization.
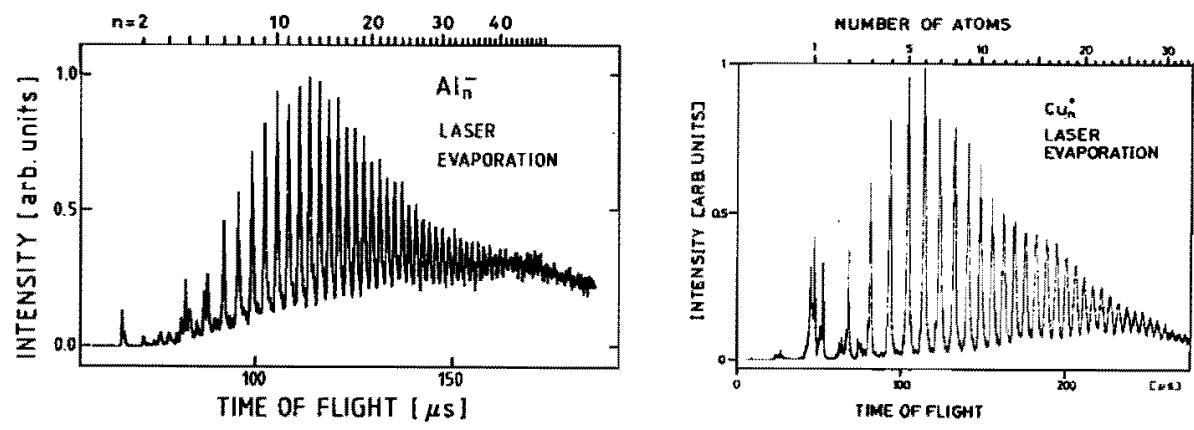

Fig. 14. Cluster spectra of cold $\mathrm{Cu}_{\mathrm{n}}^{+}$and $\mathrm{Al}_{\mathrm{n}}^{-}$obtained directly from the source

The envelope of the intensity distributions can be changed by adjusting the pulse timing, but the fine structures are not affected. For $\mathrm{Cu}_{n}^{+}$, e.g., such a spectrum is in contrast to those of hot copper cluster ions which have been heated either by ionization, or during the production by sputtering /25/: Hot $\mathrm{Cu}_{n}^{+}$ show a marked even-odd intensity distribution as well as several intensity steps in the spectrum which we have attributed to the unimolecular decomposition of excited cluster ions. Such a structure is completely missing in Fig. 14, thus proving that the temperature of $\mathrm{Cu}_{\mathrm{n}}^{+}$, obtained directly from the laser evaporation source, is at least below the bolling point.

With this experimental setup and an appropriate line shape analysis, it is possible to determine the translational temperature Ttrans: e.g., for $\mathrm{Cu}^{+}$a value of $\mathrm{T}_{\text {trans }}=6 \pm 2 \mathrm{~K}$ is obtained. Note that copper ions produced without seeding gas have a mean energy of $7 \mathrm{ev}$. 


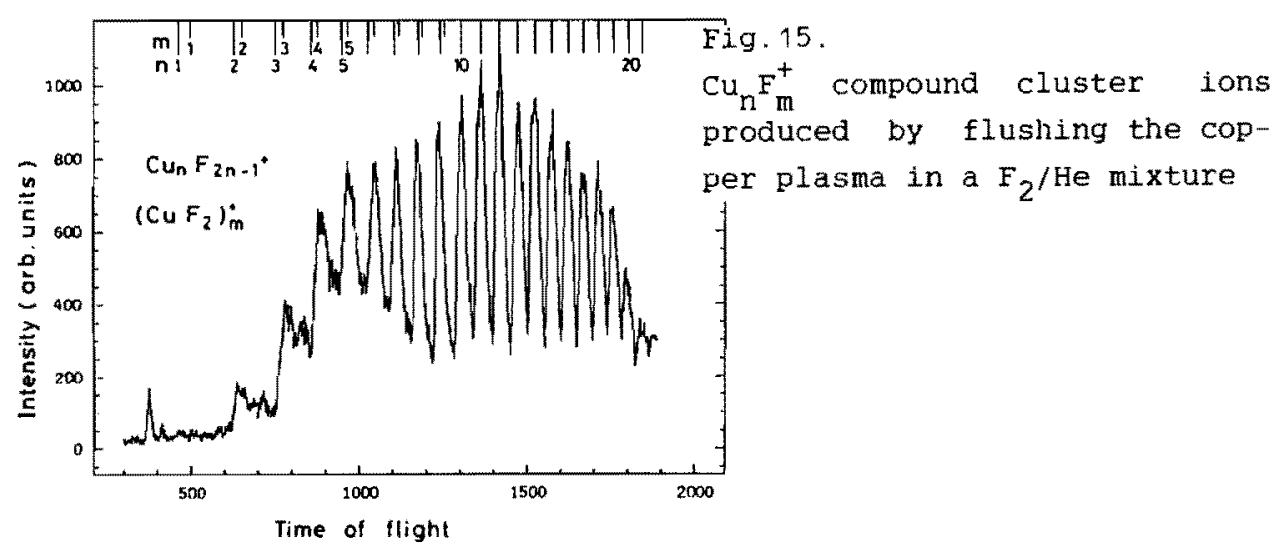

Aside from characterization of pure metal cluster ion beams, we produce metal-halogen compound clusters by adding a halogen to the seeding gas. The ion beam composition is a function of the halogen concentration. As an example Fig. 15 gives the mass spectrum of evaporated copper, seeded with $5 \% \mathrm{~F}_{2}$ in helium.

The (relatively high) $\mathrm{F}_{2}$ concentration destroys nearly any $\mathrm{Cu}_{n}^{+}$contribution. Instead, a very intense and stable $\mathrm{Cu}_{n} \mathrm{~F}_{\mathrm{m}}^{+}$signal is observed. For high $n$ the $\mathrm{Cu}_{n} \mathrm{~F}_{2 n-1}+$ sequences dominate. (CuF) ${ }_{n}{ }^{+}$appears with minor abundance only for low $n$. In contrast

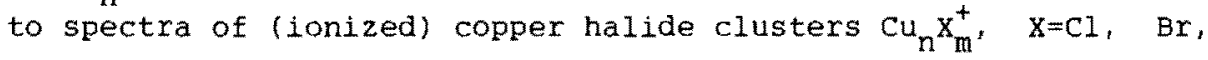
$I$, studied by MARTIN et al. /31/, low $n$ clusters have weak intensity (which is no artifact of the mass spectrometer). In addition, also in contrast to the behaviour of other copper halides, copper binds two fluorine atoms; in the strongest sequence one of the $F$ atoms is missing to give the positive charge.

At the moment it cannot be decided whether the compound cluster ions stem from laser-evaporated $\mathrm{CuF}_{2}$ species or are products of chemical reactions of copper clusters in a $F_{2}$-rich environment. The residence time of the ion pulse in the source, however, is for $\mathrm{Cu}_{n}^{+}$about twice as long as for $\mathrm{Cu}_{n} \mathrm{~F}_{\mathrm{m}}^{+}$. So the compound clusters seem not to be produced as "rigid plasma", which might be a hint to $\mathrm{CuF}_{2}$ being evaporated from the surface and growing in the extender or/and in the supersonic expansion.

\section{Photoelectron spectroscopy of $\mathrm{Al}_{\mathrm{n}}^{-}$}

Most studies of the electronic properties of larger clusters were limited to a determination of ionization potentials. Even 
such experiments might not uniquely reveal physical properties of clusters of defined size as the neutral beams are not mass selected; the nascent ions can decompose on their way to the detector and eventually prevent the ionization threshold from being assigned to the correct mass. Therefore, we decided to investigate the energy of photodetached electrons of negatively charged clusters with defined mass. Fragmentation after detachment does not influence the outcome of the experiment as the electron's energy is an image of the ion's ground state before light interaction.

only recently the groups of LINEBERGER /32/ and SMALLEY /33/ investigated the photoelectrons of $\mathrm{Cu}_{n}^{-}$. In these experiments the anions have been produced continously in a cold cathode discharge 132/ or after post-ionization of clusters in a pulsed laser vaporization source $/ 33 /$. The resulting electron affinities show a pronounced even-odd variation with decreasing amplitude for increasing $n$; strong minima are observed for $n=2,4,8 / 32 /$. A qualitatively similar $n$-dependence is seen for the intensity of sputtered $\mathrm{Cu}_{n}{ }^{-}$, see Fig.2, thus showing directly the correlation between electron binding energy and the survival of excited negative cluster ions.

We investigate the energies of photoelectrons which are detached from cold metal cluster anions. Different lines of an excimer

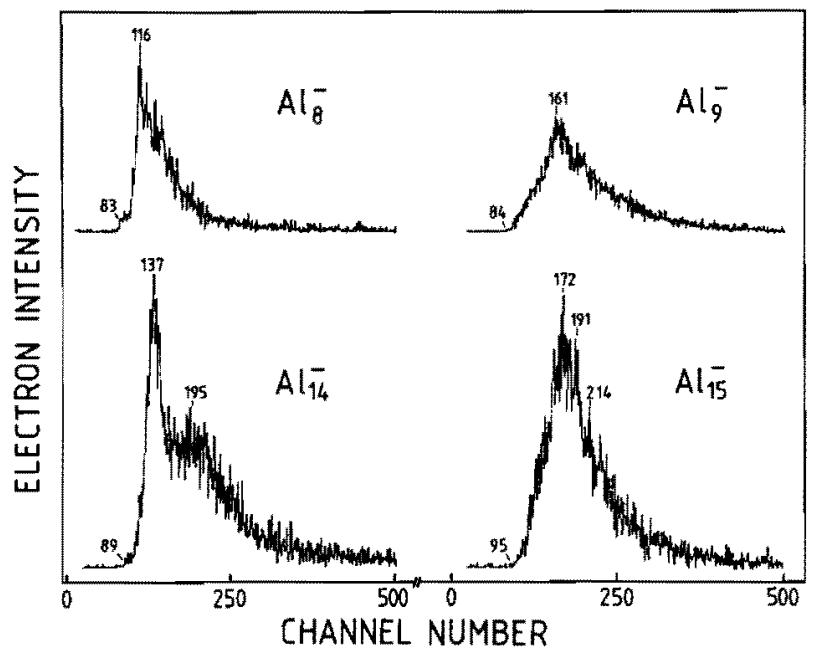

Fig. 16. Electron time-of-flight spectra of $\mathrm{Al}_{\mathrm{n}}{ }^{-}$. The intensities are in arbitrary units and not related to detachment cross sections 
laser serve as light source. As an example Fig. 16 compares some electron TOF spectra from $\mathrm{Al}_{\mathrm{n}}^{-}$.

The data are obtained at excitation energies of $3.5 \mathrm{eV}$ (XeF excimer laser) and give four typical spectra for some selected aluminium cluster ions: The electron appearance thresholds are all in the same spectral region which corresponds to upper limits of adiabatic electron affinities of $1.5 \ldots 2.0 \mathrm{eV}$. The shapes of the photoelectron bands vary irregularly with $n$. It seems that the electronic band development of aluminum clusters does not proceed continuously but rather stepwise.

A thorough discussion of our photoelectron results would go too far for the scope of this paper and will be published elsewhere 134/. Instead, we report on two general observations for $\mathrm{Al}_{n}^{-}$photoelectrons: First, the electron thresholds show an overall increase with $n$, for $2 \leq n \leq 20$, ranging from 1.4 to $2.5 \mathrm{ev}$. The latter value is still far away from the work function of aluminium ( $4.2 \mathrm{ev})$.

second, some bands show narrow lines. The origin of such structures (isomers, excited states of neutral clusters, ...) has to be clarified by additional theoretical and experimental effort.

\section{CONCLUSTON}

Mass identified cluster ions provide an excellent test ground for the investigation of the size-dependence of geometric and electronic structures. In the case of cluster production by sputtering, the unimolecular decomposition and the resulting mass spectra give important information on the thermodynamic stability and thus the binding energy. Collision induced fragmentation (CIF) of mass selected cluster ions confirms those results; in addition, the CIF cross sections reveal a (partly huge) difference between positively and negatively charged clusters and, on the other hand, between metal and semiconductor cluster ions.

Ions produced (without post-ionization) in a laser evaporation source and seeded in the or Ne are cold. Therefore the resulting mass spectra show no fine structures or "magic numbers". This might be a hint that a deviation from homogeneous mass distributions results from decompositions. 
Addition of a halogen to the seeding gas produces very intense metal fluoride cluster ion beams. Such compound clusters are cold and thus preferred for chemical reaction experiments.

The powerful tool of photoelectron spectroscopy has been applied to cold metal cluster anions. For aluminum we observe huge variations in the photoelectron band shapes, the thresholds show an overall rise with $n$.

\section{ACKNOWLEDGEMENT}

We thank Y.Y. Liu and R. Hector for helpful discussions and are obliged to W.C. Iineberger for communicating his results prior to publication. Financial support by the Deutsche Forschungsgemeinschaft is gratefully acknowledged.

\section{REFERENCES}

/ 1/ G. Staudenmaier, Rad. Eff. 18 (1973) 181

/ $2 /$ G.P. Können, A. Tip, A.E. de Vries, Rad. Eff. 21 (1974) 269 and 26 (1975) 23

W. Gerhard, H. Oechsner, Z. Phys. B 22 (1975) 41

/ 3/ R.F.K. Herzog, W.P. Poschenrieder, F.G. Satkiewicz, Rad. Eff. 18 (1973) 199

K. Wittmaack, Phys. Lett. 69A (1979) 322

For a review on cluster emission in sputtering see:

W.o. Hofer, Proc. NATO Advanced Study Institute on Erosion and Growth of Solids Stimulated by Atom and Ion Beams, eds. G. Kiriakidis et al. (M. Nijhoff, 1986) p.1

/ 4/ W. Begemann, S. Dreihöfer, K.H. Meiwes-Broer, H.O. Lutz, Z. Phys. D 3 (1986) 183

/ 5/ C.E. Klots, Z. Phys. D5 (1987) 83

/ 6/ W. Begemann, K.H. Meiwes-Broer, H.O. Lutz, Phys. Rev. Lett. 56 (1986) 2248

/ $7 /$ W. Ens, R. Beavis, and K.G. Standing, Phys. Rev. Lett. 50 (1983) 27

/ 8/ T.D. Märk, P. Scheier, K. Leiter, W. Ritter, K. Stephan, and A. Stamatovic, Int. J. Mass Spectr. Ion. Proc. 74 (1986) 281

/ $9 /$ A.W. Castleman, Jr., and R.G. Keesee, Chem. Rev. 86 (1986) 589 
/10/ B.A. Mamyrin, V.I. Karataev, D.V. Schmikk, V.A. Zagulin, Sov. Phys.-JETP 37 (1973) 45

/11/ U. Boesel, H.J. Neusser, R. Weinkauf, E.W. Schlag, J. Phys. Chem. 86 (1982) 4857

/12/ A.W. Castleman, Jr.,

o. Echt,

S. Morgan,

P.D. Das,

R.J. Stanley, Ber. Bunsenges. Phys. Chem. 89 (1985) 281

/13/ M.A. Johnson, M.L. Alexander, and W.C. Lineberger, Chem. Phys. Lett. $112(1984) 285$

/14/ Th. Leisner, O. Echt, D. Kreisle, M. Knapp, and

E. Recknagel, to be published

/15/ A. Hermann, E. Schumacher, L. Wöste, J. Chem. Phys. 68 (1978) 2327

/16/ w. Eckardt, Phys. Rev. B 29 (1984) 1558

/17/ $\mathbf{w}$. Knight, K. Clemenger, $\mathbf{W}$. de Heer, $\mathbf{W}$. Saunders, M. Chou, and M. Cohen, Phys. Rev. Lett. 52 (1984) 2141

W. Saunders, private communication

/18/ M.Y. Chou, M.L. Cohen, Phys. Lett. 113A (1986) 420

M.Y. Chou, A. Cleland, and M.L. Cohen, Solid State Comm. 52 (1984) 645

/19/ L. Wöste, P. Fayet, w. Saunders, private communications

/20/ I. Katakuse, T. Ichihara, Y. Fujita, T. Matsuo, S. Sakurai, and H. Matsuda, Int. J. Mass Spectr. Ion Proc. 69 (1986) 109

121/ A. Van Lumig and J. Reuss, Int. J. Mass Spectr. Ion Phys. 27 (1978) 197

/22/ K. Ervin, S.K. Loh, N. Aristov, and P.B. Armentrout, J. Phys. Chem. 87 (1983) 3503

/23/ M.F. Jarrold, J.E. Bower, J. Chem. Phys. 86 (1987) 3876

$124 /$ L. Hanley, S.A. Ruatta, S.L. Anderson, to be published

125/ W. Begemann, S. Dreihöfer, K.H. Meiwes-Broer, H.O. Lutz in "The Physics and Chemistry of Small Clusters", Eds. P. Jena, S. Khanna and B. Rao, NATO ASI Series, Plenum Press (1987) 269

/26/ T.H. Upton, Phys. Rev. Lett. 56 (1986) 2168

/27/ K. Raghavachari, V. Logovinsky, Phys. Rev. Lett. 26 (1987) 2853

/28/ D.E. Powers, S.G. Hansen, M.E. Geusic, D.L. Michalopoulos, and R.E. Smalley, J. Chem. Phys. 78 (1983) 2666

/29/ L.A. Bloomfield, M.E. Geusic, R.R. Freeman, and W.L. Brown, Chem. Phys. Lett. 121 (1985) 33 
/30/ P. Kruit, F.H. Read, J. Phys. E. Scientific Inst. 16(1983) 313

/31/ T.P. Martin and A. Kakizaki, J. Chem. Phys. 80 (1984) 3956

132/ D.G. Leopold, J. Ho, and W.C. Lineberger, J. Chem. Phys. 86 (1987) 1715

/33/ 0. Cheshnovsky, P.J. Brucat, S. Yang, C.L. Pettiette, M.J. Craycraft, and R.E. Smalley, in "The Physics and Chemistry of Small Clusters", Eds. P. Jena, S. Khanna and B. Rao, NATO ASI Series, Plenum Press (1987)

/34/ G. Ganteför, K.H. Meiwes-Broer, H.O. Lutz, to be published (1987) 